\title{
Quality Randomized Clinical Trials
} of Topical Diabetic Foot Ulcer Healing Agents

\author{
Laura L. Bolton* \\ Department of Surgery, Rutgers Robert Wood Johnson University Medical School, New Brunswick, New Jersey.
}

Significance: Diabetic foot ulcers (DFU) significantly add to global economic, social, and clinical burdens. Healing a DFU fast and well limits complications that can lead to lower extremity amputation, morbidity, and mortality.

Recent Advances: Many promising topical DFU healing agents have been studied in randomized clinical trials (RCT), but only one, becaplermin, has been cleared for this use by the United States Food and Drug Administration (FDA). Critical Issues: This critical review of DFU topical healing RCTs summarizes issues identified in their design and conduct, highlighting ways to improve study quality so researchers can increase the likelihood of RCT success in propelling effective topical DFU healing agents toward clinical use. Key issues include (1) inadequate sample size, (2) risk of bias, (3) irrelevant or unreported inclusion criteria, (4) substandard outcome measures, (5) unmatched group characteristics that predict nonhealing at baseline, (6) unequal or uncontrolled concurrent interventions or standard of care, (7) heterogeneous subject or DFU samples (8) unblinded allocation, treatment, or outcome measures, or (9) inadequate follow-up for clinical relevance. These can add bias or unexplained variability to RCT outcomes, limiting clinical or statistical significance and accuracy of results.

Future Directions: This critical review summarizes ways to overcome these deficiencies to optimize DFU clinical trial design and conduct. It provides a blueprint for future excellence in RCTs testing safety and efficacy of topical DFU healing agents and smoothing the path to their clinical use.

\section{SCOPE AND SIGNIFICANCE}

DEVELOPING A TOPICAL DIABETIC foot ulcer (DFU) healing agent requires care. Once preclinical efficacy, safety, purity, consistency, and delivery are established, the agent's safety and efficacy must be supported with rigorous, relevant, credible randomized clinical trials (RCTs) meeting recognized standards. One flaw can invalidate RCT results, reducing the sponsor's credibility at great costs of money and time that few can afford. This review summarizes recognized standards of RCT design and conduct to answer the safety and efficacy questions typically raised by health care professionals and authorities responsible for regulatory clearance and reimbursement of topical DFU healing agents.

\section{TRANSLATIONAL RELEVANCE}

Years of preclinical translational research lay scientific foundations for active topical DFU healing agents, generating hypotheses, guiding development, and supporting the agent's safety and efficacy in treating models of diabetic wounds, but diabetic mice don't walk on neuropathic foot ulcers as patients often do on their DFUs. Only well designed, well-conducted,

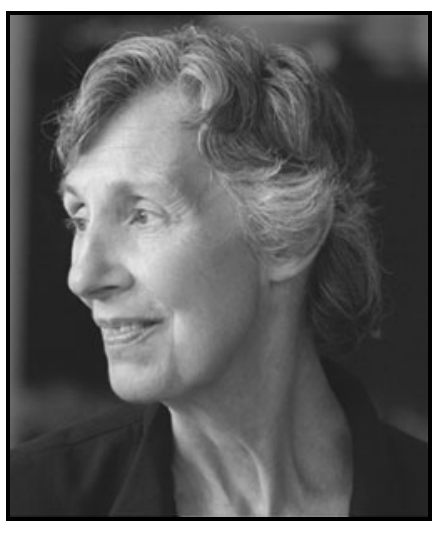

Laura L. Bolton, PhD

Submitted for publication June 15, 2014. Accepted in revised form August 4, 2014.

*Correspondence: 15 Franklyn Place, Metuchen, NJ 08840-2307 (e-mail: Ilbolton@gmail.com). 
prospective double-blind RCTs can determine without bias whether the agent works safely on clinical DFUs and improves clinical outcomes more than an identical placebo used with identical good standard of care (SOC) procedures. This review highlights DFU RCT quality methods to optimize the transition from preclinical to clinical product development.

\section{CLINICAL RELEVANCE}

A relevant, well designed, conducted, analyzed, and reported RCT can determine safety and efficacy of a topical DFU healing agent in improving clinical, economic, or patient-oriented outcomes beyond those afforded by best evidence-based standardized care. Its outcomes can support regulatory clearance, reimbursement, and clinical education about its use on DFU patients similar to those studied.

DFU RCTs widely vary in quality of design, conduct, and outcomes reporting, raising difficulties in discerning the comparative value an agent adds to DFU management. This review summarizes elements of topical DFU healing agent RCT excellence encouraging improvement in study design, conduct, analysis, and reporting.

\section{BACKGROUND \\ Problem}

DFUs are a serious source of morbidity and mortality associated with the global diabetes epidemic ${ }^{1}$ challenging patients, clinicians, health care systems, and nations. ${ }^{2}$ DFU healing restores a patients' quality of life ${ }^{3}$ and reduces likelihood of complications that can lead to lower extremity amputation and ensuing 5-year mortality rates exceeding those of some of the most deadly cancers. ${ }^{4,5}$

Many promising topical DFU healing agents in clinical development aim to heal DFU before patients enter this lethal progression. These greatly needed breakthroughs pass from hypothesis through preclinical verification and clinical observation culminating in rigorous Phase II and Phase III prospective RCTs proving safety and efficacy before they are cleared for clinical use to help heal DFUs. Once a safe, effective agent is cleared for use, its supporting RCTs can further help encourage clinical use through education and reimbursement. A flawed early RCT can jeopardize development of a promising DFU healing agent, with rarely a second chance to prove its worth. Rigorous standards of RCT quality have been published, ${ }^{6}$ but are not always used in design or conduct of DFU RCTs.
Problems arise when researchers apply the less rigorous methods of pre-RCT observations to the conduct of Phase II and III RCTs, resulting in their low quality. One can hardly expect a flawed study to reveal unbiased facts about an agent's safety or efficacy. Biasing either subject allocation to groups, treatment methods, outcomes measurement, or reporting may reveal what one wants to hear and improve the likelihood of temporary funding, but it does not lead to sustainable clinical benefit, funding, or profit.

The history of wound healing is replete with low quality "RCTs" that falsely augured healing efficacy others could not replicate. These wasted time, energy, and funds that could have been used better to guide wound care into a scientifically enlightened era of practice, informing wound care patients, professionals, industry, and governments about agents that work safely so all flourish.

Recognized principles (Table 1) support RCT high evidence quality ${ }^{7-11}$ for Phase II and III studies of topical active agents for healing DFU. RCTs applying these principles may be expected to yield more accurate, less biased information about efficacy, safety, or patient acceptability, the essential pillars supporting clinical and commercial success. Recognized RCT quality scoring systems are based on these principles, such as the JADAD score, ${ }^{12}$ which accents blinding methods or the Cochrane evaluation system, ${ }^{13}$ which emphasizes study bias. Rigorously applying the principles in Table 1 will yield high quality RCT evidence to inform clinical practice and earn the respect of scientific, clinical, regulatory, and reimbursement authorities.

\section{Objective}

This critical review summarizes published flaws from prior DFU clinical trials and highlights ways to avoid them by adhering to essentials of RCT design, conduct, analysis, and reporting for Phase II and III clinical studies testing safety and efficacy of topical DFU healing agents.

\section{DISCUSSION OF FINDINGS AND RELEVANT LITERATURE}

Once a concept has passed the observation stage and has demonstrated preclinical safety and efficacy testing on relevant models, it is ready for high quality RCTs that test its safety and efficacy on clinical subjects. These RCTs are so costly and laborious that it pays to do them right to obtain clear answers about safe and effective dose administration on the first trial. Table 1 provides an essential "To do" list for designing, conducting, and reporting a high quality RCT that will pass scrutiny of 
Table 1. Hallmarks of a high quality randomized clinical trial testing efficacy and safety of topical diabetic foot ulcer healing agents

\begin{tabular}{|c|c|}
\hline Study Feature Location in Publication & Hallmarks of High Quality RCT Design, Conduct, or Reporting ${ }^{7-11}$ for a Topical DFU Healing Agent \\
\hline Abstract & $\begin{array}{l}\text { Clear, accurate, succinct summary of objective, design, methods, including setting, subjects, outcome } \\
\text { measures, significant results, and conclusions }\end{array}$ \\
\hline Relevant clinical need & $\begin{array}{l}\text { Clinical need to improve DFU healing in the population from which the study sample will be drawn establishing } \\
\text { relevance }\end{array}$ \\
\hline Rationale for study & Evidence that this topical agent may safely, effectively heal DFU \\
\hline Objective & Clearly states subjects, treatment, setting, and outcomes explored. \\
\hline Methods & All stated below match Objective and are clearly, completely described \\
\hline Trial design & Prospective, randomized, parallel (or factorial, randomized block etc.) clinical trial \\
\hline Study sample & $\begin{array}{l}\text { Subject inclusion and exclusion criteria are clearly stated and adhered to } \\
\text { Sample size is sufficient for statistical power } \geq 80 \% \text { for primary outcome } \\
\text { Groups are similar on baseline parameters affecting the primary healing outcome }\end{array}$ \\
\hline $\begin{array}{l}\text { Procedures and observations (data collection and } \\
\text { disposition) }\end{array}$ & $\begin{array}{l}\text { Subject, care provider, and outcome evaluator are blinded to treatment, as feasible. } \\
\text { Treatment procedures are clearly described to enable study replication, stating who did what to whom in what } \\
\text { setting, how often and by when }\end{array}$ \\
\hline Interventions and SOC & $\begin{array}{l}\text { Clearly described, ideally varying only in active agent (e.g., identical placebo or vehicle or sham control, with } \\
\text { the same SOC applied consistently to all subjects). If not, authors clearly state why this was not done and } \\
\text { include it among study limitations discussed. }\end{array}$ \\
\hline Outcomes & $\begin{array}{l}\text { Clinically relevant, prespecified valid, reliably measured primary and secondary outcomes are ideally evaluated } \\
\text { by an assessor blinded to treatment assigned. }\end{array}$ \\
\hline Statistical analysis & $\begin{array}{l}\text { Prespecified planned statistical comparisons for all stated outcomes are described, including any planned } \\
\text { interim analyses and/or study stopping points. } \\
\text { Baseline comparability of subjects and wounds in all compared treatment groups are described clearly and } \\
\text { added subset analyses, ad hoc or adjusted analyses. }\end{array}$ \\
\hline Results & Record or report all results that were measured clearly and accurately \\
\hline Conclusions & Significant results are accurately described without embellishment or omission \\
\hline Acknowledgment, access & All source(s) of support, funding, sponsorship, and accessibility are clearly stated. \\
\hline Funding or sponsorship & Funding source(s) and influence(s) on data acquisition, analysis or reporting are cited. \\
\hline Trial accessibility & $\begin{array}{l}\text { Provide trial registration number, name of trial registry if applicable, and source(s) for further information about } \\
\text { the RCT or its data. }\end{array}$ \\
\hline
\end{tabular}

ITT, intent-to-treat sample of all subjects randomized to and receiving at least one treatment; PP, per protocol sample of all subjects adhering to the study protocol per specified conditions; RCT, randomized clinical trial.

clinical decision makers, guideline developers, literature reviewers, and regulatory and reimbursement authorities. Such RCTs are usually designed to answer the question "Can this intervention improve outcome(s) beyond effects of its vehicle used with the best available standard of care"14,15 Fig. 1 illustrates how a clearly stated question generates study design and conduct for DFU topical healing agent RCTs.

This question requires an RCT meeting criteria in Table 1 for an unbiased answer because random subject allocation avoids biasing results by assur- ing that each subject has an equal likelihood of being assigned to receive each intervention.

To help match groups at baseline on factors that affect the likelihood of healing, one may also stratify random assignment on one or more of these factors. For example, separate computerized randomization allocation sequences could be used to assign subjects with a baseline target DFU area of $0.5-4 \mathrm{~cm}^{2}$ or $>4-6 \mathrm{~cm}^{2}$ as increasing DFU area increases risk of delayed DFU healing. ${ }^{16}$

Another key to minimizing bias is assuring that all treatments and outcomes evaluations are conducted 


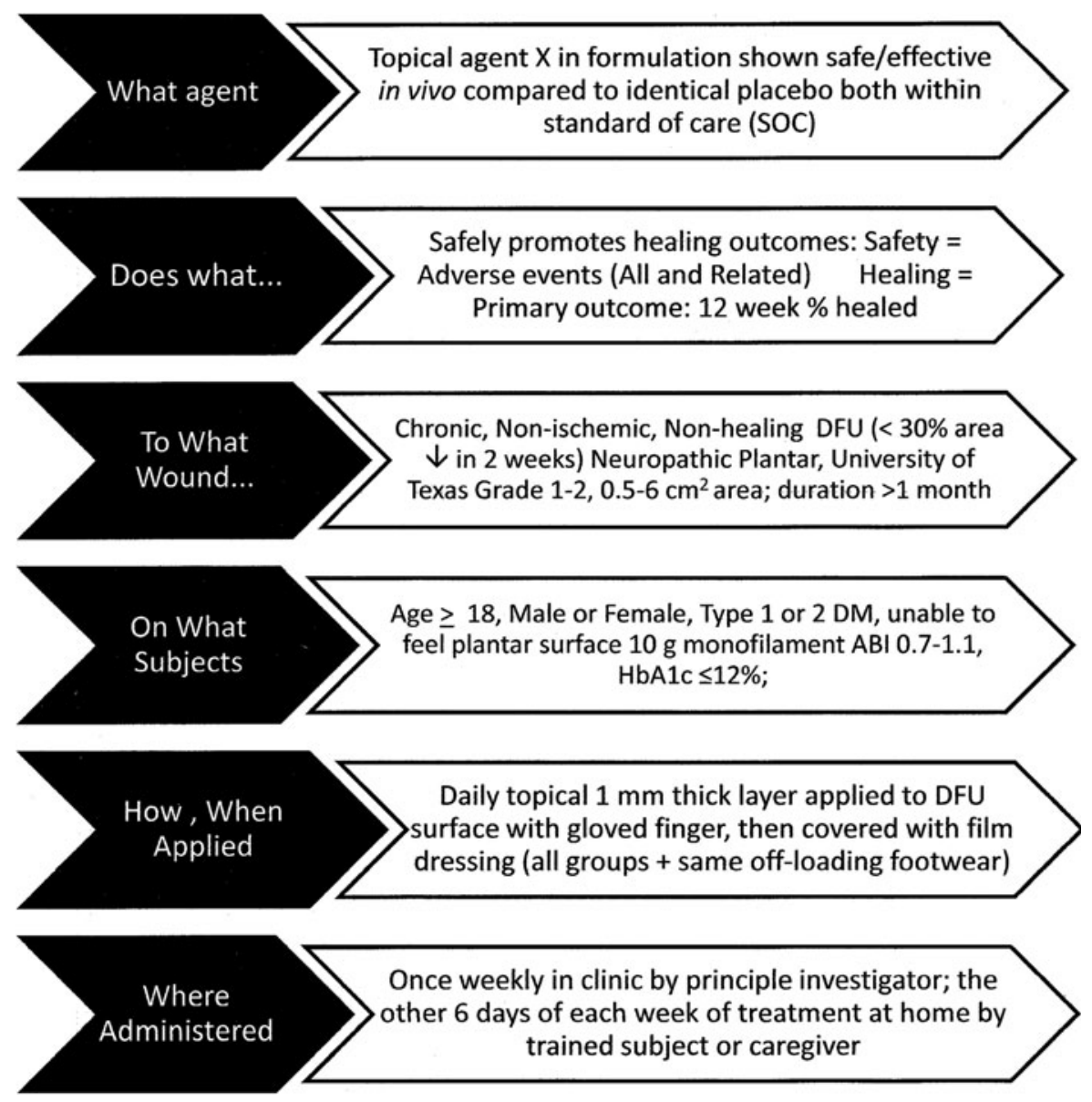

Figure 1. Good study design flows from a hypothesis-generated question thoughtfully crafted to meet patient, professional, institution, regulatory, and reimbursement needs. This fictitious example illustrates the question, “Does Agent X safely promote healing of Wagner Grade 1, 2 plantar DFU in outpatients with a chronic nonischemic, nonhealing, neuropathic DFU?"

and received without knowledge of what intervention the subject is receiving, also known as "blinding." Though often difficult, especially for active agents that noticeably differ from controls, it is worth the effort to arrange for the evaluator of wound outcomes, and if possible, the patients, to be blinded to treatments assigned, so that only the person applying the agent knows the treatment assigned.

Randomization and blinding are only two general tactics to help assure that RCTs answer their questions clearly and honestly, fostering development of safe, effective products that support clinical outcomes, patient benefit, product registration and clinical education initiatives. This review examines how DFU topical healing RCTs measure up to recognized RCT quality metrics, and summarizes practical techniques from DFU literature to enhance RCT quality.

\section{Methods}

Topical DFU healing agent RCT literature was reviewed to identify common DFU RCT flaws to encourage future improved quality and efficiency of clinical research. MEDLINE database searches were conducted from inception to February 1, 2014, for the combined terms "diabetic foot ulcer randomized topical" with and without the added term "quality." The ClinicalTrials.gov database was searched up to the same date for all RCTs investigating topical healing agents tested on DFU for healing efficacy and safety. Focused Google Scholar searches added information about specific RCT design and outcomes as needed.

Prospective parallel-group RCTs in any setting or country were included if they reported complete DFU healing results for subjects with Type 1 or 2 diabetes mellitus and a Wagner ${ }^{17}$ or University of Texas $^{18}$ Grade 1 or 2 DFU treated with a topical vulnerary agent for at least 2 weeks with outcomes reported at least 8 weeks after the first treatment. Studies of topical agents used on DFU were excluded if they did not report healing outcomes of a topically administered active healing agent. Studies of physical stimuli such as offloading modalities, 
hyperbaric oxygen, negative pressure or electrical or electromagnetic stimulation were excluded.

\section{Results}

The MEDLNE and ClincalTrials.gov literature searches identified 291 publications, including 80 citations addressing 41 RCTs of effects of topical agents applied to a DFU. Five systematic reviews ${ }^{19-23}$ described common flaws or issues observed in relevant RCTs (Table 2). Actions DFU researchers can take to help assure the quality and credibility of RCTs include the following:

1. Use adequate sample size. In Phase II RCTs at least 20 subjects per group helps to assure generality. For pivotal Phase III RCTs, sample size estimates can be found in statistical tables ${ }^{24}$ or other statistical sources. Ideally, choose a sample size sufficient to achieve statistical power of at least $80 \%$ with a 5\%Type 1 (alpha) error of falsely concluding healing efficacy (statistically rejecting the null hypothesis) for a clinically important difference in the primary outcome for example, a 15 percentage point difference between $50 \%$ of active and $35 \%$ of control subjects completely healed at 12 weeks.

Some regulatory authorities require a twotailed Type 1 error in this calculation, assuming equal likelihood of benefit or harm of the active agent in Phase III studies, inflating the sample sizes required. While this may seem absurd if healing outcome(s) sig- nificantly favored the active agent in its Phase II studies, the inflated sample sizes help assure that related safety issues are addressed on the package insert.

2. Randomize allocation to groups without bias. Using unbiased allocation at the point of subject assignment to treatment groups, such as computerized random number generators accessed via computer or by unsealing an opaque envelope or by the flip of a fair coin, assures that each subject has an equal likelihood of being assigned to all treatment groups.

3. Maintain blinded treatment and evaluation. Ideally all investigators, subjects, care providers, and those evaluating results should remain unaware of treatments assigned until the database is locked. If the treatment and controls are noticeably different, at least outcome evaluators, and to the extent possible, patients, should remain blinded to treatment during the study.

Actions (2) and (3) avoid risk of allocation, treatment, evaluation, and other forms of bias that can raise participant and caregiver expectations and affect results. If this is not feasible, reasons for this limitation should be clearly described in all public communications. That it "is not ethical" to engage in such double- or triple-blinded studies is not a valid reason to avoid such blinding. This assumes the agent's efficacy before proven.

Table 2. Diabetic foot ulcer topical treatment study flaws described in published reviews of diabetic foot ulcer literature

\begin{tabular}{|c|c|c|}
\hline Source & Study Findings & Most Common Flaws Described \\
\hline Dumville et al. ${ }^{19}$ & $\begin{array}{l}\text { In order hydrocolloid dressings had highest odds of being } \\
\text { associated with best DFU healing outcomes followed by } \\
\text { hydrogel, then foam topical dressings }\end{array}$ & $\begin{array}{l}\text { Only } 3 \text { of } 15 \text { RCTs reported DFU healing time } \\
\text { Mainly less severe, less complex DFU were studied } \\
\text { Most studies were at high or unclear risk of bias } \\
\text { Most studies had small sample sizes leading to low certainty of outcomes }\end{array}$ \\
\hline Gottrup and Apelqvist ${ }^{20}$ & $\begin{array}{l}\text { There is limited high quality evidence on topical DFU dressings } \\
\text { and healing agents and an urgent need to increase the quality } \\
\text { of DFU clinical studies }\end{array}$ & $\begin{array}{l}\text { Most studies had inadequate sample size, short follow-up, nonrandom } \\
\text { allocation to treatment arms, nonblinded assessment of outcomes, poor } \\
\text { description of controls, with concurrent interventions unmatched on } \\
\text { groups of heterogeneous samples of subjects and DFU }\end{array}$ \\
\hline Shaw et al. ${ }^{21}$ & $\begin{array}{l}\text { One moderate quality RCT supported a healing effect of topical } \\
\text { phenytoin on DFU }\end{array}$ & $\begin{array}{l}\text { Most articles failed to adequately describe randomization, treatment } \\
\text { allocation, and blinding techniques }\end{array}$ \\
\hline Buchberger et al. ${ }^{22}$ & $\begin{array}{l}\text { There may be an advantage for add-on therapy with EGF or } \\
\text { becaplermin growth factors in diabetic foot ulcers for complete } \\
\text { wound closure and time to complete wound healing outcomes }\end{array}$ & $\begin{array}{l}\text { Differences in standard wound care complicated the comparison of study } \\
\text { results. Many RCTs had small to very small sample sizes and other } \\
\text { methodological flaws with high potential for bias. The duration of } \\
\text { treatment and follow-up examinations was not long enough to assess } \\
\text { the sustainability of healing and surveillance of ulcer recurrences or } \\
\text { treatment-related adverse events like the development of malignancy }\end{array}$ \\
\hline Edwards and Stapley ${ }^{23}$ & $\begin{array}{l}\text { RCTs reported DFU healing outcomes using surgical, larval, } \\
\text { autolytic, or gauze debriding interventions. Only topical } \\
\text { application of autolytic hydrogels resulted in faster DFU } \\
\text { healing compared with gauze }\end{array}$ & $\begin{array}{l}\text { Most studies had inadequate power to find clinically significant effects, } \\
\text { with unreported inclusion and exclusion criteria for example, neuropathy } \\
\text { Study details for example, setting or primary pathology of enrolled subjects } \\
\text { or random sequence generation were often unreported } \\
\text { Subject assignment to group (allocation) was often not blinded, creating } \\
\text { potential bias }\end{array}$ \\
\hline
\end{tabular}


4. Use and clearly report relevant inclusion criteria, such as neuropathy (e.g., with sensation measured using Semmes-Weinstein monofilaments at five specified plantar sites) so that subjects are documented to be relevant to the indication planned for the agent's use. Using selected inclusion criteria known to predict healing to stratify group assignment on enrollment can help avoid imbalanced groups at analysis.

Alternatively, these baseline inclusion measurements can support planned analyses that clarify treatment effects independently of baseline group differences. Two examples of frequently unreported or misinterpreted inclusion criteria discussed below are DFU nonhealing status and depth.

4a. Include nonhealing DFU. Most DFU heal within 12 weeks with good SOC treatment and consistent offloading ${ }^{25}$ and may not need the intervention studied. Enrolling these healing DFU decreases likelihood of finding statistically significant treatment effects on healing outcomes because of high control DFU healing rates.

DFU RCTs with vague inclusion criteria for "nonhealing” DFU, for example, DFU of over 1 month duration without complete healing may all heal quickly without differentiating important treatment effects. Including DFU that reduce in surface area less than 53\% when treated with best practice SOC for 4 weeks $^{26,27}$ (or less than 25-30\% during a prerandomization 2 -week screening period) focuses on DFU unlikely to heal with a good SOC during 12-20 weeks.

4b. Baseline depth is another important predictor of DFU healing ${ }^{28}$ to consider among inclusion criteria. Standardized Wagner or University of Texas grading scales confound DFU depth with abscess, infection and/or ischemia. Neither of these DFU grading systems clearly differentiate full- from partial-thickness DFU, which substantially differ in their healing processes: the deeper the wound the more granulation tissue is required to fill it ${ }^{29}$ before it re-epithelizes. As a result, full-thickness chronic ulcers require twice the time to heal as similar area partialthickness ulcers in the same cohort. ${ }^{30}$

Options for depth measurement include recording full- or partial-thickness depth at baseline or monitoring DFU millimeters of deepest depth at baseline and at each DFU measurement during the RCT. The latter measure of DFU depth reduction can reflect significant responses to topical therapy before DFU area reduction differences occur. $^{31,32}$ Carefully considered inclusion criteria like these can increase the likelihood of identifying an effective healing agent.

5. Use recognized, standardized outcome measures. Though it is tempting to use early surrogate measures of healing, reducing clinical trial length and cost, some regulatory authorities still require measures involving complete healing to clear a topical agent's DFU healing claim.

Two healing outcomes recognized by the United States Food and Drug Administration $(\text { FDA })^{6}$ are (1) time to complete healing of the study DFU with no visible wound or residue, verified as completely closed 2 weeks later and (2) percent of study DFU completely healed, similarly verified 2 weeks after initial healing, following a standardized interval between first treatment and outcome measurement, usually10 or 12 weeks.

It may be more appropriate to call the latter measure "percent durably closed" because it combines initial biologic wound closure and 2 -week durability of wound closure. This measure, while clinically important, blurs the distinction between these two biologically independent healing outcomes and misses opportunities to observe topical healing agents' effects on them in important clinical trials. It further confuses estimates of healing durability, raising the question of when to start counting weeks that the wound remained closed.

Other measures monitoring biologic healing progress, such as percent reduction in area after 4 to12 weeks on study or time to $50 \%$ reduction in area or time to achieve a wound bed adequate for surgical closure may be worthwhile secondary outcomes important to patients and clinicians, but these are currently viewed by regulatory authorities as inadequate to support a DFU healing claim.

These important outcomes include patientreported quality of life and wound-related pain intensity and duration, frequency and amounts of antibiotic or pain medication requirements, length of hospital stay, time for patient to resume normal daily activities, percent of graft take or incidence of clearly documented wound complications such as necrosis, in- 
fections, scar contractures, elevation or color, or time to achieve negative wound bacterial cultures or to prepare the wound bed for surgical closure or grafting.

6. Match groups on patient and wound characteristics that predict DFU healing at baseline. This contributes to homogeneity and comparability of samples of subjects and DFU. Stratified random assignment can help assure that groups are similar at baseline on key healing predictors such as DFU baseline area less than $4 \mathrm{~cm}^{2}$ or 4 to $6 \mathrm{~cm}^{2} 16$ or whether the DFU was on a normal or delayed healing path $^{20}$ as illustrated in the Enrollment section of the example CONSORT Flow Diagram (Fig. 2) created for a fictitious DFU RCT from examples in the literature.

Stratifying on more than one baseline characteristic can become complicated at the point of subject randomization increasing the likelihood of errors in subject assignment. In case chance assigns more subjects with larger or longer duration ulcers to one group than another causing unbalanced groups on that variable, it can help to plan an appropriate multiple regression or covariate analysis to calculate healing effects of the topical agent that are independent of the unbalanced variable.

7. Rigorously control all concurrent interventions, so that all groups in all centers receive the same SOC and identical interventions except for differing concentrations of the one agent being studied. Figure 3 lists interventions identified as SOC elements ${ }^{6,14,15}$ with examples supported by the DFU literature reviewed. ${ }^{23,33-36}$

Some elements of an SOC can perform double duty. For example hydrogels used to maintain a moist environment for autolytic debridement can accelerate DFU healing ${ }^{23}$ while moist healing with hydrocolloid dressings has been associated with a lower incidence of DFU infection than traditional gauze dressings. ${ }^{25}$ Use of another moist

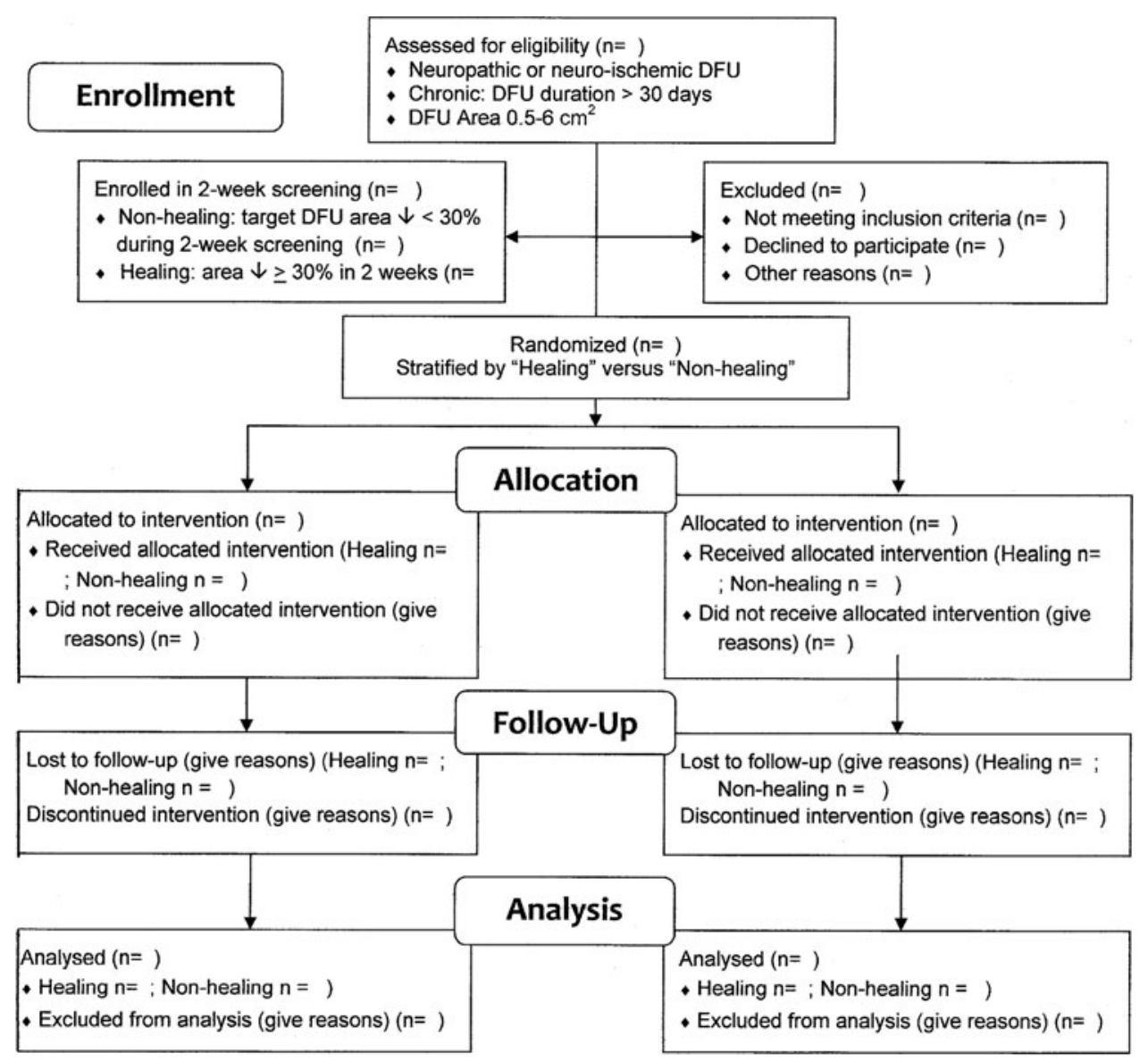

Figure 2. Example CONSORT Study Flow Diagram of a randomized clinical trial (RCT) of efficacy and safety of a topical diabetic foot ulcer (DFU) healing agent with planned subset analyses for healing and nonhealing DFU. (Derived from a template accessed at www.consort-statement.org/downloads) 


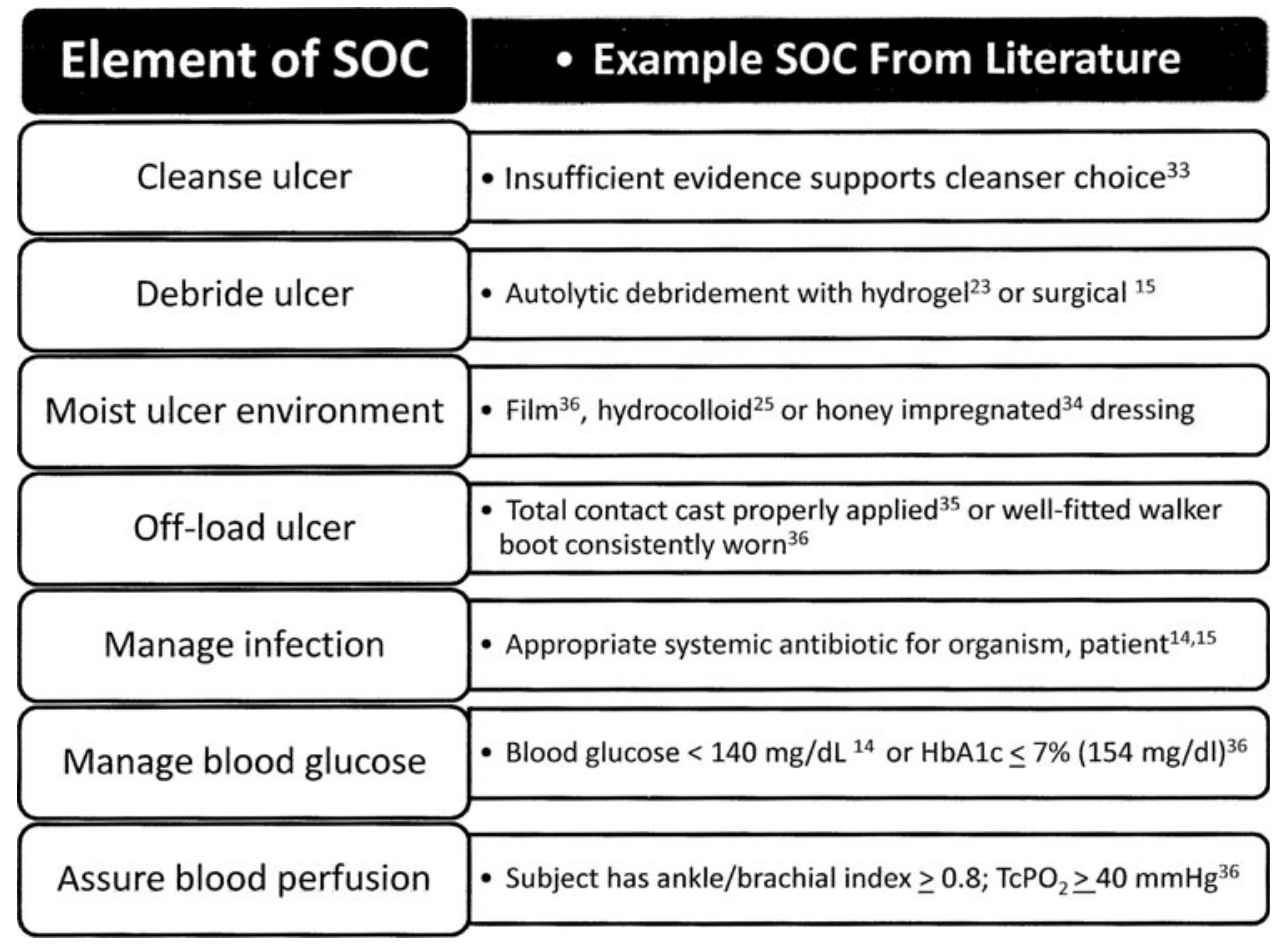

Figure 3. Example elements of a diabetic foot ulcer standard of care (SOC) based on recommendations of the International Working Group on the Diabetic Foot practical guidelines, ${ }^{14}$ the American Diabetes Association consensus statement ${ }^{15}$ and the FDA Guidance for conducting clinical trials on chronic wounds and burns. ${ }^{6}$

wound healing intervention, honey-impregnated dressings, led to faster DFU healing and earlier bacteria-free wound cultures without systemic antibiotic use compared in a RCT to saline gauze dressed controls. ${ }^{34}$

While it is difficult to design a single protocol that addresses all patient and wound needs, a good protocol can include standardized intervention variations that address common conditions. For example, a topical healing agent may be sealed over the DFU with a film or hydrocolloid dressing with addition of an absorbent dressing overlay during periods of high exudate to prevent leakage. Such a standardized method of managing excess exudate, consistently documented in case report forms, could maintain moist wound healing while preventing exudate and topical treatment leakage between treatment intervals for all subjects.

Undocumented or unplanned variations among active or control interventions allowed to vary among groups can confound RCT results, making it difficult to distinguish effects of the active agent from those of the concurrent interventions. Variations in concurrent interventions in cases required by clinical need should be clearly documented and ana- lyzed to help clarify these potentially confounding effects.

8. Conduct adequate follow-up to assure clinical relevance of the primary healing outcome. Follow-up observations assess safety and durability of healing following treatment usually for at least 1 month in individuals with a DFU. Ideally healed DFU remain healed as long as good SOC is practiced, but some subjects with diabetes may need help to follow good SOC practices. ${ }^{14}$

Adhering to the principles of quality RCT design and conduct outlined in Table 1 would address each of these issues, improving DFU RCT design, conduct, and reporting to generate quality, credible wound care evidence that propels promising DFU healing agents forward. This high quality research has the potential to generate well-researched breakthroughs in DFU management. Global patients, clinicians, payers, product developers, and governments all gain if wound care researchers routinely apply these rigorous principles of RCT quality.

Exploring how current evidence measures up to the criteria in Table 1, the literature search identified 41 unique RCTs that reported recognized DFU healing outcomes. It is not meaningful to compare best results identified for the outcome, 


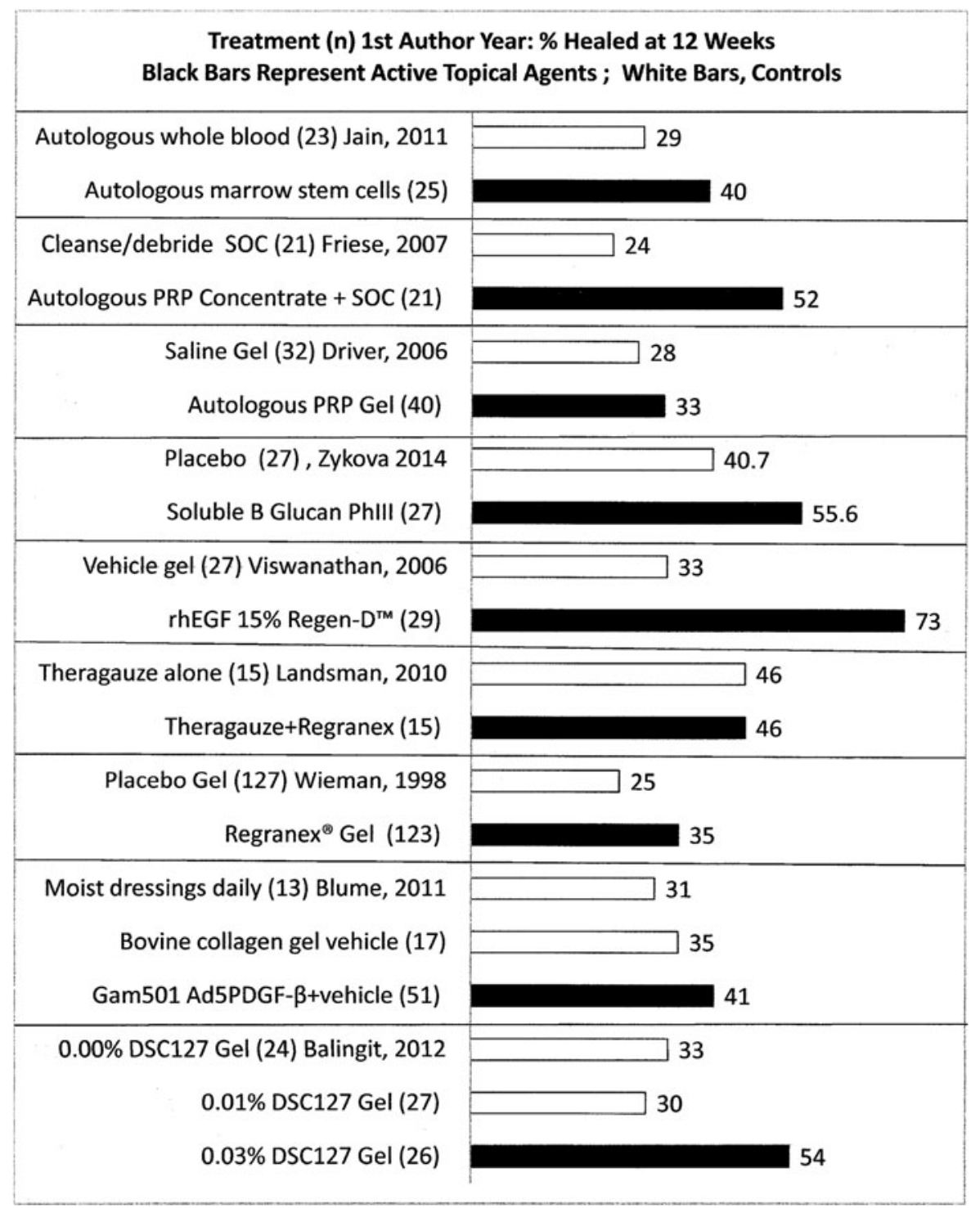

Figure 4. It is not meaningful to compare a simple outcome like percent of subjects completely healed at 12 weeks across these nine RCTs ${ }^{36-44}$ due to study differences in analyses, blinding of allocation, treatment or evaluation, subject and ulcer variables, and variability in concomitant interventions such as offloading or other parameters.

"percent of subjects completely healed at 12 weeks" presented in Fig. $4^{36-44}$ because these RCTs varied in SOC procedures (e.g., off-loading varying from crutches or wheelchairs to walker boots held in place with bandaging), length of follow-up (none to 12 weeks) and subject baseline characteristics (e.g., duration of diabetes and degree of metabolic control) and DFU inclusion criteria (ulcer duration, depth, and area on enrollment, healing or nonhealing status, Wagner or University of Texas Grade 1 or $1-2$ ). In one study within-trial comparisons challenged interpretation because the SOC for control subjects differed from that of subjects receiving the active agent. ${ }^{35}$ Among the
41 RCTs reporting recognized outcomes, 21 (51\%) were double-blind studies, with six more (15\%) blinded to either patient or outcome evaluator. Nearly half of these trials had high potential for bias because those evaluating results knew which treatment each subject received. Of the nine RCTs in Fig. 4, six were conducted double-blinded to treatment and evaluation and three ${ }^{35-37}$ were not blinded. The net result is a relatively weak foundation of DFU RCT evidence on which to base clinical decisions. Patients, clinicians, and reimbursement authorities deserve stronger science to inform their decisions about managing wounds with such devastating consequences. 


\section{SUMMARY}

Many topical DFU treatment RCTs are conducted with quality, consistency, and rigor, but quality improvement is needed for most RCTs, especially in the use of adequate sample sizes to detect clinically significant outcome differences, standardizing blinding, conduct and reporting of randomized enrollment, procedures, outcomes, and follow-up measures and assuring uniform adjunctive and concurrent care across patients and centers.

\section{ACKNOWLEDGMENTS AND FUNDING SOURCES}

The author gratefully acknowledges the United States Agency for Healthcare Research and Quality, the Cochrane Collaboration and Guidelines International Network for their training and online resources that greatly contributed to this article. No funding was received to write this article.

\section{AUTHOR DISCLOSURE AND GHOSTWRITING}

No competing financial interests exist. The content of this article was expressly written by the author listed. No ghostwriters were used to write this article

\section{ABOUT THE AUTHOR}

Laura Bolton is Adjunct Assoc. Professor in the Department of Surgery at Rutgers Robert Wood Johnson University Medical School, New Brunswick, New Jersey and the co-chair of Association for the Advancement of Wound Care Guideline Task Force, Malvern, Pennsylvania.

\section{TAKE-HOME MESSAGES}

- Ask the right question, designing the study to include clinically relevant patients with clearly specified comorbidity limits each with one nonhealing study DFU of relevant area, duration, and depth.

- Any study worth doing is worth doing well. Use best DFU practice SOC continued during treatment with a clinically relevant study duration and follow-up.

- Rigorously control all concurrent interventions throughout the study, holding all treatment variables constant except the healing agent studied.

- For RCTs of healing efficacy, assure that groups are similar at baseline on key DFU healing predictors and consistently measure and report recognized outcomes meeting patient and clinical needs of importance to regulatory authorities in the countries where the agent will be used.

- Wield statistics wisely to generate the minimum sample size to find clinically relevant healing differences and plan statistical analyses to adjust for inadvertent group imbalances.

- Assure that the topical DFU healing agent under study improves clinically relevant outcomes beyond best DFU SOC, consistent offloading with a total contact cast (TCC) or "instant TCC" 45 and moisture-retentive dressings, $34,36,46$ not outdated care such as saline gauze or crutches for offloading. Moist gauze rarely keeps wounds moist more than $4 \mathrm{~h}^{47}$ providing substandard care ${ }^{48,49}$ associated with increased pain and infection rates and delayed healing in acute and chronic wounds. ${ }^{50-52}$

- Avoid bias by using appropriate blinded random allocation to treatments, with patients, caregivers, investigators, and evaluators all blinded to treatment groups ${ }^{53}$ until the database is locked.

- Consider applying these principles to RCT design, conduct, and reporting for other wound care interventions, as they reinforce recent consensus statements applicable to a wide range of wound indications. ${ }^{53}$

\section{REFERENCES}

1. Gupta SK, Singh SK. Diabetic foot: a continuing challenge. Adv Exp Med Biol 2012;771:123-138.

2. Sen CK, Gordillo GM, Roy S, et al. Human skin wounds: a major and snowballing threat to public health and the economy. Wound Repair Regen 2009;17:763-771.

3. Armstrong DG, Lavery LA, Wrobel JS, Vileikyte L. Quality of life in healing diabetic wounds: does the end justify the means? J Foot Ankle Surg 2008;47:278-282.

4. Boulton AJ, Vileikyte L, Ragnarson-Tennvall G, Apelqvist J. The global burden of diabetic foot disease. Lancet 2005;366:1719-1724.
5. Armstrong DG, Wrobel J, Robbins JM. Guest editorial: are diabetes related wounds and amputations worse than cancer? Int Wound J 2007:4:286-287.

6. US Department of Health and Human Services, Food and Drug Administration. Guidance for Industry: Chronic cutaneous ulcer and burn wounds: Developing products for treatment. June 2000. www.fda/cber/ guidelines.htm (last accessed November 2, 2011).

7. Jaeschke R, Guyatt G, Meade M. Evidence-based practice: what it is, why we need it. Adv Wound Care 1998;11:214-218.

8. Gray M, Beitz J, Colwell J, et al. Evidence-based nursing practice II. JWOCN 2004;31:53-61.
9. Agency for Healthcare Research and Quality Evidence Report/Technology Assessment Number 47 Systems to Rate the Strength of Scientific Evidence. (AHRO Publication No. 02-E016.)

10. Bolton LL, Dotson P, Kerstein M. Controlled clinical trials versus case studies: Why wound care professionals need to know the difference. Chapter 7 In: Krasner DL, Rodeheaver GT, Sibbald RG, eds. Chronic Wound Care: A Clinical Source Book for Healthcare Professionals. 4th ed. Malvern, PA: HMP Communications, 2007:57-66.

11. CONSORT Group. Consolidated Standards of Reporting Trials. CONSORT statement checklist and 
study flow diagram. www.consort-statement.org/ downloads (last accessed May 15, 2014).

12. Jadad AR, Moore RA, Carroll D, et al. Assessing the quality of reports of randomized clinical trials: is blinding necessary? Controlled Clin. Trials 1996;17:1-12.

13. Higgins JPT, Altman DG, Sterne JAC. Chapter 8: Assessing risk of bias in included studies. In: Higgins JPT, Green S, eds. Cochrane Handbook for Systematic Reviews of Interventions Version 5.1.0 (updated March 2011). The Cochrane Collaboration, 2011. www.cochrane-handbook.org (accessed March 15, 2014).

14. Bakker K, Apelqvist J, Schaper NC; International Working Group on Diabetic Foot Editorial Board. Practical guidelines on the management and prevention of the diabetic foot 2011. Diabetes Metab Res Rev 2012;28 Suppl 1:225-231.

15. American Diabetes Associaton. Consensus development conference on diabetic foot wound care. Diabetes Care 1999;22:1354-1360.

16. Margolis DJ, Allen-Taylor L, Hoffstad O, Berlin JA. Diabetic neuropathic foot ulcers: the association of wound size, wound duration and wound grade on healing. Diabetes Care 2002;25:1835-1839.

17. Wagner FW Jr. The dysvascular foot: a system for diagnosis and treatment. Foot Ankle 1981;2:64-122.

18. Oyibo SO, Jude EB, Tarawneh I, Nguyen HC, Harkless LB, Boulton AJ. A comparison of two diabetic foot ulcer classification systems: the Wagner and the University of Texas wound classification systems. Diabetes Care 2001;24:84-88.

19. Dumville JC, Soares MO, O'Meara S, Cullum N. Systematic review and mixed treatment comparison: dressings to heal diabetic foot ulcers. Diabetologia 2012;55:1902-1910.

20. Gottrup F, Apelqvist J. Present and new techniques and devices in the treatment of DFU: a critical review of evidence. Diabetes Metab Res Rev 2012;28 Suppl 1:64-71.

21. Shaw J, Hughes CM, Lagan KM, Bell PM. The clinical effect of topical phenytoin on wound healing: a systematic review. Br J Dermatol 2007;157:997-1004.

22. Buchberger B, Follmann M, Freyer D, Huppertz H, Ehm A, Wasem J. The importance of growth factors for the treatment of chronic wounds in the case of diabetic foot ulcers. GMS Health Technol Assess 2010;6:Doc12.

23. Edwards J, Stapley S. Debridement of diabetic foot ulcers. Cochrane Database Syst Rev 2010;1:CD003556.

24. Dixon WJ, Massey FJ. Introduction to Statistical Analysis. 4th ed. Table A-12f Sample sizes for two sample binomial tests. New York: McGraw-Hill Publishing Company, 1983:570-571.

25. Boulton A, Meneses P, Ennis WJ. Diabetic foot ulcers: a framework for prevention and care. Wound Rep Reg 1999;7:7-16.

26. Sheehan P, Jones P, Caselli A, Giurini J, Veves A. Percent change in wound area of diabetic foot ulcers over a 4-week period is a robust predictor of complete healing in a 12-week prospective trial. Diabetes Care 2003;26:1879-1882.

27. Snyder RJ, Cardinal M, Dauphinée DM, Stavosky J. A post-hoc analysis of reduction in diabetic foot ulcer size at 4 weeks as a predictor of healing by 12 weeks. Ostomy Wound Manage 2010;56:44-50.
28. Ince P, Abbas ZG, Lutale JK, et al. Use of the SINBAD classification system and score in comparing outcome of foot ulcer management on three continents. Diabetes Care 2008;31:964-967.

29. Bolton L, Vasko A, Monte K. Quantification of wound healing. Chapter 17 In: Schwindt D, Maibach $\mathrm{H}$, eds. Cutaneous Biometrics. New York: Kluwer Academic/Plenum Publishers, 2000:205-219

30. Bolton L, McNees P, van Rijswijk $L$, et al.; the Wound Outcomes Study Group. Wound-healing outcomes using standardized assessment and care in clinical practice. JWOCN 2004;31:65-71.

31. Jude E, Apelqvist I, Spraul M, Martini J. Prospective randomized controlled study of nonischaemic diabetic foot ulcers dressed with Hydrofiber ${ }^{\circledR}$ containing ionic silver or calcium alginate dressings. Diabetes Med 2007;24:280-288.

32. Reyzelman A, Armstrong DG, Foreman $P$, et al. Prospective, randomized, open-label, comparative study of Hydrofiber ${ }^{\circledR}$ dressing with silver and saline-moistened gauze in the management of diabetic foot ulcers. Proceedings Canadian Association of Wound Care, Montreal, November 12-15, 2005.

33. Fernandez R, Griffiths R. Water for wound cleansing. Cochrane Database Syst Rev 2012;2:CD003861.

34. Kamaratos AV, Tzirogiannis KN, Iraklianou SA, Panoutsopoulos GI, Kanellos IE, Melidonis Al. Manuka honey-impregnated dressings in the treatment of neuropathic diabetic foot ulcers. Int Wound J 2012;9:1-7

35. Armstrong DG, Nguyen HC, Lavery LA, van Schie $\mathrm{CH}$, Boulton AJ, Harkless LB. Off-loading the diabetic foot wound: a randomized clinical trial. Diabetes Care 2001;24:1019-1022. Erratum in: Diabetes Care 2001;24:1509.

36. Balingit PP, Armstrong DG, Reyzelman AM, et al. NorLeu(3)-A(1-7) stimulation of diabetic foot ulcer healing: Results of a randomized, parallel-group, double-blind, placebo-controlled phase 2 clinical trial. Wound Repair Regen 2012;20:482-490.

37. Blume P, Driver VR, Tallis AJ, et al. Formulated collagen gel accelerates healing rate immediately after application in patients with diabetic neuropathic foot ulcers. Wound Rep Reg 2011;19:302-308.

38. Driver VR, Hanft J, Fylling CP, Beriou JM; Autologel Diabetic Foot Ulcer Study Group. A prospective, randomized, controlled trial of autologous plateletrich plasma gel for the treatment of diabetic foot ulcers. Ostomy Wound Manage 2006;52:68-87.

39. Friese $G$, Herten M, Scherbaum WA. The use of autologous platelet concentrate activated by autologous thrombin $(\mathrm{APC}+)$ is effective and safe in the treatment of chronic diabetic foot ulcers-a randomized controlled trial. Paper presented at: Fifth International Symposium on the Diabetic Foot; May 9-12, 2007, 2007; Noordwijkerhout, The Netherlands.

40. Jain P, Perakath B, Jesudason MR, Nayak S. The effect of autologous bone marrow-derived cells on healing chronic lower extremity wounds: results of a randomized controlled study. Ostomy Wound Manage 2011;57:38-44.

41. Landsman A, Agnew P, Parish L, Joseph R, Galiano RD. Diabetic foot ulcers treated with becaplermin and TheraGauze, a moisture-controlling smart dressing: a randomized, multicenter, prospective analysis. J Am Podiatr Med Assoc 2010;100:155-160.
42. Viswanathan V, Pendsey S, Sekar N, Murthy GSR. A phase III study to evaluate the safety and efficacy of recombinant human epidermal growth factor (REGEN-D_ 150) in healing diabetic foot ulcers. Wounds 2006;18:186-196.

43. Zykova SN, Balandina KA, Vorkhobina NV, Kuznetsova AV, Engstad R, Zykova TA. Macrophage stimulating agent soluble yeast $\beta$-1,3/1,6-glucan as a topical treatment of diabetic foot and leg ulcers: a randomized double blind, placebo controlled phase II study. J Diabetes Invest 2014;5:392-399.

44. Wieman TJ, Smiell JM, Su Y. Efficacy and safety of a topical gel formulation of recombinant human platelet-derived growth factor-BB (becaplermin) in patients with chronic neuropathic diabetic ulcers. A phase III randomized placebo-controlled doubleblind study. Diabetes Care 1998;21:822-827.

45. Armstrong DG, Lavery LA, Wu S, Boulton AJ. Evaluation of removable and irremovable cast walkers in the healing of diabetic foot wounds: a randomized controlled trial. Diabetes Care 2005;28:551-554.

46. Bolton LL. Evidence-based report card: operational definition of moist wound healing. JWOCN 2007;34: 23-29.

47. Colwell J, Foreman MD, Trotter JP. A Comparison of the efficacy and cost-effectiveness of two methods of managing pressure ulcers. Decubitus 1993;6:28-36.

48. National Institute for Clinical Excellence (N.I.C.E.). Guidance on the use of debriding agents and specialist wound care clinics for difficult to heal surgical wounds. Technology Appraisal Guidance No. 24, April 2001.

49. Cordrey R. Gauze, impregnated gauzes, and contact layers. In: Sen CK, ed. Advances in Wound Care. New Rochelle, NY: Mary Ann Liebert, Inc. 2010;1:120-125.

50. Brölmann FE, Eskes AM, Goslings JC, et al.; REMBRANDT study group. Randomized clinical trial of donor-site wound dressings after split-skin grafting. Br J Surg 2013;100:619-627.

51. Wiechula R. The use of moist wound-healing dressings in the management of split-thickness skin graft donor sites: a systematic review. Int $\mathrm{J}$ Nurs Pract 2003;9:S9-S17.

52. Hutchinson JJ, McGuckin M. Occlusive dressings: a microbiologic and clinical review. Amer J Infec Control 1990;18:257-268.

53. Serena T, Bates-Jensen B, Carter MJ, et al. Consensus principles for wound care research obtained using a Delphi process. Wound Repair Regen 2012; 20:284-293.

Abbreviations and Acronyms
$\mathrm{DFU}=$ diabetic foot ulcer
$\mathrm{FDA}=$ Food and Drug Administration
$\mathrm{ITT}=$ intent-to-treat sample of all subjects
randomized to and receiving at least
one treatment
$\mathrm{PP}=$ per protocol sample of all subjects
adhering to the study protocol per
specified conditions
$\mathrm{RCT}=$ randomized clinical trial
$\mathrm{SOC}=$ standard of care
$\mathrm{TCC}=$ total contact cast

